\title{
Generalized Noise Resonance: Using Noise for Signal Enhancement
}

\author{
Ferran Martorell ${ }^{a}$, Mark D. McDonnell ${ }^{b}$, Derek Abbott $^{b}$ and Antonio Rubio ${ }^{a}$ \\ ${ }^{a}$ Univ. Politècnica de Catalunya (DEE), Ed.C-4 Jordi Girona 1-3 08034, Barcelona, Spain; \\ ${ }^{b}$ Centre for Biomedical Engineering (CBME) and School of Electrical \& Electronic \\ Engineering, The University of Adelaide, SA 5005, Adelaide, Australia
}

\begin{abstract}
Noise is a key factor in information processing systems. This fact will be even more critical in new technologies, as dimensions continue to scale down. New design methodologies tolerant to or even taking advantage of noise need to be considered. In this work the possibility of using stochastic resonance (SR) in electronic circuits is studied. We demonstrate the validity of nearly any kind of perturbing signal in producing a noise resonance, thus extending the stochastic resonance concept. In this paper we have explored stochastic, chaotic, deterministic and coupled noise perturbations. The relationship between input signal and input noise amplitude on the noise resonance regime is analyzed, providing a rule for operation under this situation. Finally, we present a simulation study demonstrating that noise resonance is robust to non-ideal behaviors of non-linear devices. All three facts allow direct use of generalized noise resonance (GNR) in electronic circuits.
\end{abstract}

Keywords: noise, chaos, suprathreshold stochastic resonance, stochastic resonance, nonlinear circuits, low SNR signal processing, generalized noise resonance, chaotically induced noise resonance

\section{INTRODUCTION}

Noise is present in all systems in any of its possible forms. Fluctuations are inherent in physical systems, and cannot be fully avoided. Furthermore, electrical, chemical or mechanical activity can produce fluctuations in corresponding variables that can disturb nearby systems. It is possible to reduce the magnitude of fluctuations in some cases, but undesired perturbations persist and are ubiquitous. Biological systems have evolved to be either immune to noise or to take advantage of it. Several studies show how biological sensory systems use noise to improve their sensitivity, ${ }^{1,2}$ how sensory neurons deal with information in a noisy environment ${ }^{3}$ where signal-to-ratio is $0 \mathrm{~dB}$, and even how neurons can use noise to achieve a better working dynamic range. ${ }^{4}$

However, in man-made systems, noise is considered a limiting factor in performance. In communication equipment noise in channel and in receiver circuits limits the minimum detectable signal. Also, in mixed integrated circuits noise from digital circuitry degrades performance of nearby analog circuitry. Traditional design methodologies consider noise as a nuisance, providing techniques to reduce its effect on circuits. The importance of noise in circuit design depends on the characteristics of the technology. As technology advances, smaller circuits are manufactured and undesired interactions between each component increases. With increasing miniaturization, the supply voltage level decreases and thus the signal amplitude decreases approaching inherent noise levels. In newly emerging nanotechnologies that utilize, for example, single electron transistors (SETs), nanotubes or molecular electronics, noise will be more critical as signal levels and dimensions will be scaled down.

As noise cannot be avoided and its influence will increase, as technology scales down, it is necessary to look for a different strategy to deal with it as nature does. Some applications show that noise can be used to advantage. For example, in measurement, adding noise and averaging afterwards produces a resolution improvement. ${ }^{5}$ Moreover, in ADC/DAC applications dithering also permits extra resolution and noise may

Further author information: (Send correspondence to F.M.)

F.M.: E-mail: ferranm@eel.upc.es, Telephone: +34-93-4017-485

M.D.M.: E-mail: mmcdonne@eleceng.adelaide.edu.au, Telephone: +61-8-8303-6296

D.A.: E-mail: dabbott@eleceng.adelaide.edu.au, Telephone: +61-8-8303-5748

A.R: E-mail: rubio@eel.upc.es, Telephone: +34-93-4017-485 
avoid undesired instabilities, ${ }^{6}$ fluctuations and vibrations can be used to obtain energy, ${ }^{7}$ or, more recently, stochastic resonance (SR) in nanotube FETs can be used to improve signal detection in nanoantennas. ${ }^{8}$

Our work studies stochastic resonance phenomenona with the objective of using noise in electronic systems. The work is based in a single threshold device, ${ }^{9}$ producing SR. ${ }^{10,11}$ Furthermore, we consider an array of $N$ parallel thresholding elements, producing suprathreshold stochastic resonance (SSR). ${ }^{12}$ Section 2 presents the ideal structure and derives a simple mathematical analysis for the system that allows estimation of the system's output and error. Section 3 describes our simulation setup and measures of noise resonance. In Section 4, we perform several simulations demonstrating the resonance due to noise amplitude. Our work starts with well known stochastic signals, but it extends the scope to other kinds of perturbing signals such as chaotic signals, noise coupled in complex systems or deterministic signals. In all cases the resonance is present and we call this generalized noise resonance or GNR. Also the relationship between input and noise amplitude, on noise resonance, is presented providing a design rule for operating circuits in this regime. Finally, Section 5 analyzes how nonideal characteristics of comparators affect the performance of the resonance showing that the improvement of information transfer from input to output by noise is robust to physical device characteristics.

\section{SYSTEM ANALYSIS}

\subsection{Structure description}

The work that follows is based on a single threshold device. We also extend this to an array of thresholding elements, where the outputs are summed and normalized. Figure 1 shows the structure. Basic characteristics of the threshold element are presented in Fig. 1a. The threshold element input, $S_{i}(t)$, is composed of the input signal, $X(t)$, with an added perturbation, $\eta_{i}(t)$. The signal is compared with a fixed threshold $T$, resulting in the output $\zeta_{i}(t)$ according to (1).

$$
\zeta_{i}(t)=\left\{\begin{array}{cl}
D / 2 & \text { if } X(t)+\eta_{i}(t)>T \\
-D / 2 & \text { otherwise }
\end{array}\right.
$$

Figure 1b shows the scheme for a multi-threshold system. In this case $N$ threshold elements, shown as blocks in Fig. 1a, are placed in parallel with their inputs connected to the system's input signal. Individual outputs are combined, giving the system's output $\varphi(t)$ as $(2)$ indicates.

$$
\varphi(t)=\frac{\sum_{i}^{N}\left(\zeta_{i}(t)\right)}{N}
$$

Its expected value is the same as the individual signals, but with a variance $\sigma^{2}$ equal to the individual variance reduced by a factor $1 / N$ as Equation (3) indicates. Expected value and variance are conditioned to $X(t)$ as they show the performance of the system in relation to input signal.

$$
E[\varphi(t) \mid X(t)]=E\left[\zeta_{i}(t) \mid X(t)\right] \quad \sigma^{2}[\varphi(t) \mid X(t)]=\frac{1}{N} \sigma^{2}\left[\zeta_{i}(t) \mid X(t)\right] .
$$

\subsection{Analysis}

The system's output is completely described by determining the expected value and variance for one threshold device as shown in (3). Both values can be expressed in terms of the probabilities of each device being in a high or low state, as (4) and (5) indicate. They depend on the probability density function (pdf) of the perturbing signal through the relation in (6). So the system is fully defined by the distribution of values of the perturbing signal,

$$
\begin{gathered}
E\left[\zeta_{i}(t) \mid X(t)\right]=\frac{D}{2}\left[1-P\left(\eta_{i} \leq T-X(t)\right)\right]-\frac{D}{2}\left[P\left(\eta_{i} \leq T-X(t)\right)\right] \\
\sigma^{2}\left[\zeta_{i}(t) \mid X(t)\right]=D^{2}\left[1-P\left(\eta_{i} \leq T-X(t)\right)\right]\left[P\left(\eta_{i} \leq T-X(t)\right)\right] \\
P\left(\eta_{i} \leq T-X(t)\right)=\int_{-\infty}^{T-X(t)} p\left(\eta_{i}\right) d \eta_{i}
\end{gathered}
$$



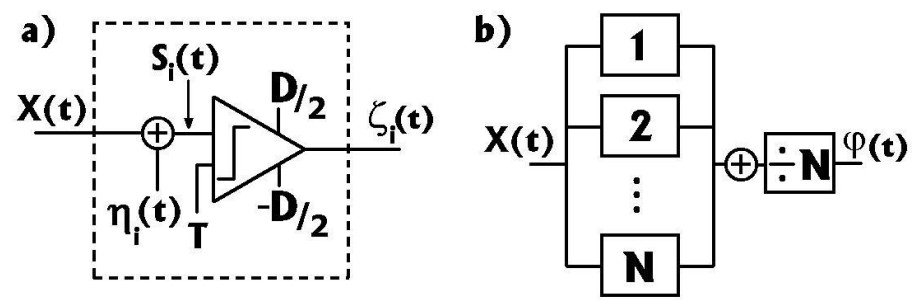

Figure 1. Ideal structure of the simulated circuits. a) Basic threshold element structure. It consists of an ideal comparator with threshold $T$ and output values $D / 2$ and $-D / 2$. Its actual input, $S_{i}(t)$, is composed by the ideal input, $X(t)$, with an added interfering signal $\eta_{i}(t)$. b) System structure for multiple cells: an input array composed by $N$ identical elements with all their outputs averaged to provide the system's output $\varphi(t)$.

where $p(\eta)$ is a probability density function (pdf). When considering a stochastic perturbing signal with uniform distribution between $-A$ and $A$, the system's function is described as (7). The mean output is the same input signal translated from the input range (defined by the noise values, $2 A$ ) to the output margin (source levels, $D)$. The threshold value produces an offset, also translated from the input to the output range. The mapping between input and output intervals can be considered as a gain on the input signal of value $D / 2 A$, for a uniform distribution, and a function over it related to the perturbing pdf. The error at the output is maximal when the input signal equals the threshold value. Its magnitude depends on the output margin and the number of elements in the input array. So the error has a range of values between $D^{2} / 4 N$ and 0 , when $X(t)$ is at a distance $A$ of the threshold and noise cannot produce any transition,

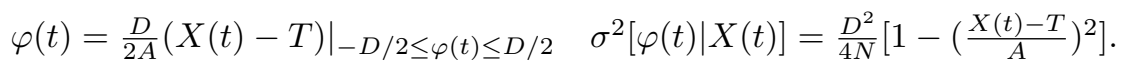

The calculation of the expected value does not depend on the nature of the perturbing signal. All signals can be characterized by the probabilities of appearance of each value and using the resulting pdf to derive the system's expected output. Non-linear functions are modified by noise, due to the effect called noise induced linearization.

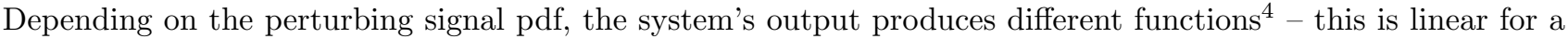
uniform distribution, error function for normal distribution and so on.

Output variance depends on the nature of the perturbing signal. Perturbing signals modify the effective threshold of each cell. In an ideal situation, thresholds can be placed equally distributed along the range of the input values. In this case, the system behaves as a normal ADC with $N+1$ levels along the output range with a quantization error of $D /(N+1)$. When real noise is considered it is possible to have repeated $\eta_{i}$ values in more than one element. This situation reduces the possible quantization levels and consequently increases the output variance. So depending on the perturbing signal characteristics the maximum variance will be bounded between values in (8) corresponding to a stochastic signal and to quantization error. These upper bounds are valid only if signals are spatially uncorrelated otherwise the average error is $D / 2$ (for no noise or equal noise value in each device) when all system works as a unique threshold cell $(N=1)$,

$$
\sigma_{\max \text { random }}^{2}=\frac{D^{2}}{4 N} \quad \sigma_{\max \text { equally distributed }}^{2}=\frac{D}{12(N+1)}
$$

The system function can be understood as the interaction of non-linear elements with the perturbing signal to produce transference of information from input to output and an averaging of the output to eliminate part of the noise and induce noise linearization. Filtering can be carried out in the temporal and spatial domains, depending on the system's characteristics. When noise is common to all threshold elements, no spatial information can distinguish the input signal from noise and using more than one element is useless. In this case the SR effect with frequency filtering must be used. However when perturbing signals are different, for each element and at each time instant, in most of the threshold devices spatial averaging may be used. In this situation interference at any frequency can be reduced (even with the same frequency, see section 4.3) without limiting the signal bandwidth, where the maximum frequency depends on the response time of devices. This is the same effect used in array processing to detect different sources depending on their relative position in space. ${ }^{13}$ 


\section{SIMULATION DESCRIPTION}

The system configuration and input signal are kept constant for all simulations to allow direct comparison of results. The threshold devices have threshold levels set to $0 \mathrm{~V}$ and supply values of -1 and $1 \mathrm{~V}(D=2 \mathrm{~V})$. The input signal is a sinusoidal waveform with frequency $10 \mathrm{~Hz}$, amplitude $0.25 \mathrm{~V}$ and offset $-0.4 \mathrm{~V}$. The frequency is chosen arbitrarily as it has no effect on system function as shown in (4) and in. ${ }^{9}$ Amplitude and offset values have also been selected arbitrarily, as Section 4.5 gives their influence in system performance.

The simulation process consists of evaluating Equation (1) for each cell and computing the average of all cell outputs. Due to this process it is necessary to define a time step $\Delta t$. This value should be small enough to provide sufficient signal resolution for the simulations - if periodic waveforms are used as perturbing signals at least 500 points per period must be considered to provide each element with a different value. In all the simulations $\Delta t$ is $0.1 \mathrm{~ms}$ except in Section 4.3 where $0.01 \mathrm{~ms}$ and in Section 4.4 where $1 \mathrm{~ms}$ are considered. The interfering signals are generated by an algorithm, so each point in the sequence is associated with a discrete-time simulation step. As a consequence white noise in the frequency band $1 / \Delta t$ is generated. We use this technique for the stochastic, chaotic and coupled noise simulations.

Noise resonance plots are presented using a SNR measure, due to its broad use and its simplicity. For a sine wave input signal this is valid. To calculate SNR, the FFT of each temporal sequence is produced using Matlab functions. To ensure accuracy, temporal simulations last for $2 \mathrm{~s}$ (i.e. 20 periods of input signal). Signal power is calculated as the peak at $10 \mathrm{~Hz}$ (i.e. the input signal frequency). DC and signal power are removed and the noise power is calculated by integrating over all remaining frequencies. The SNR value is then the ratio of both values. The presented graphs are for for both SR and SSR phenomena. They present SNR in dB at the system's output against the standard deviation $\sigma$, in $\mathrm{V}_{\mathrm{rms}}$, of the noise signals at the input.

\section{NOISE RESONANCE}

Since the discovery of stochastic resonance, ${ }^{11}$ non-linear bistable elements with added stochastic noise have received much attention. It has been demonstrated that resonance appears for periodic and aperiodic ${ }^{14}$ input signals, that the phenomenon appears for a broad range of noise distributions ${ }^{15}$ and that signals can be either subthreshold (in a single element system) or suprathreshold ${ }^{12,16}$ (in an array system). Also it has been shown that biological systems use this phenomenon to improve neural response. ${ }^{1,2}$ Nervous systems and, in particular, brain information processes use noise to improve their functions. ${ }^{4}$ There is no a priori reason as to why the noise in such systems has to be purely stochastic in nature. Moreover, extending the system analysis to other forms of noise is quite tractable. In this section, noise induced resonance produced by a range of perturbing signals with very distinct characteristics is presented - thus generalizing the concept of stochastic resonance. Stochastic, chaotic, deterministic and capacitively coupled signals are all considered.

\subsection{Stochastic signals}

Uncorrelated stochastic signals with different distributions have been the main setup of SR and SSR. As these are well established phenomena, the stochastic resonance plots will provide a benchmark for this work. Stochastic signals in simulation are usually obtained using a pseudo-random generator algorithm. In this paper we have used the rand function provided by Matlab. Its algorithm has a period of $2^{1492}$, enough to provide valid results in presented simulations. Distributions considered are the uniform (the simplest) and the normal (the most common in physical processes). Other distributions have been studied in other works ${ }^{15}$ with satisfactory results. Figure 2 presents our stochastic resonance plots. It can be easily observed that both phenomena, SR (Fig. 2 left) and SSR (Fig. 2 right, with $N=5,50$ and 500 elements in the input array) appear.

\subsection{Chaotic signals}

Several studies show that brain dynamics present chaotic activity. ${ }^{17}$ One of the goals of SR work is to understand how neurons can process information in a very noisy environment $\left(0 \mathrm{~dB}\right.$ SNR, for example $\left.{ }^{3}\right)$. If chaotic patterns appear in brain activity it is possible that chaotic perturbations affect neurons. Recently it has been shown that chaos can provide better results than stochastic noise in systems - for example, genetic algorithms driven by a chaotic sequence can converge more efficiently. ${ }^{18}$ Thus, comparing the performance in the SR regime is 

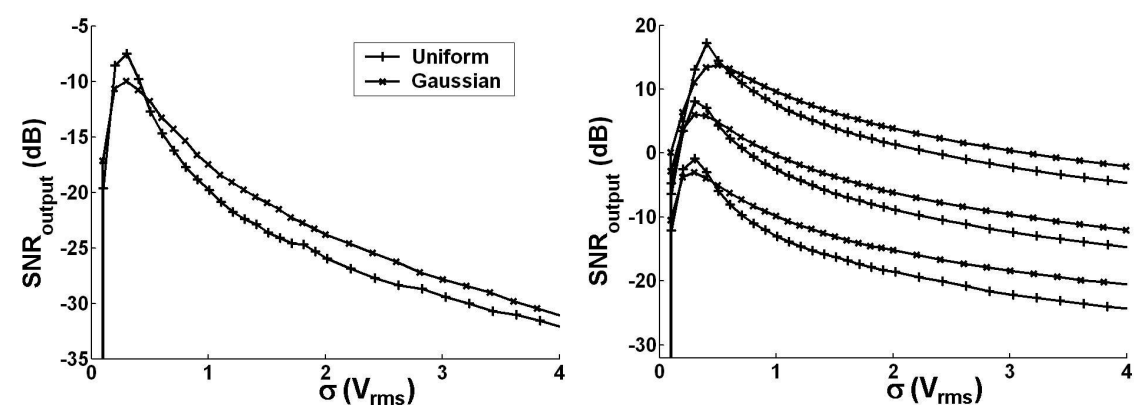

Figure 2. SNR at the system output as a function of the standard deviation of the perturbing signal in $\mathrm{V}_{\mathrm{rms}}$ in systems driven by stochastic signals (uniform and normal distributions). The left hand plot corresponds to the single threshold element. The right hand plot is for an array with $N=5,50$ and 500, from the lower to higher sets of curves. The input signal is a sinusoid with amplitude $0.25 \mathrm{~V}$ and offset $-0.4 \mathrm{~V}$ and frequency $10 \mathrm{~Hz}$. Threshold elements configuration is $D=2$ and $T=0$ according to Figure 1 .
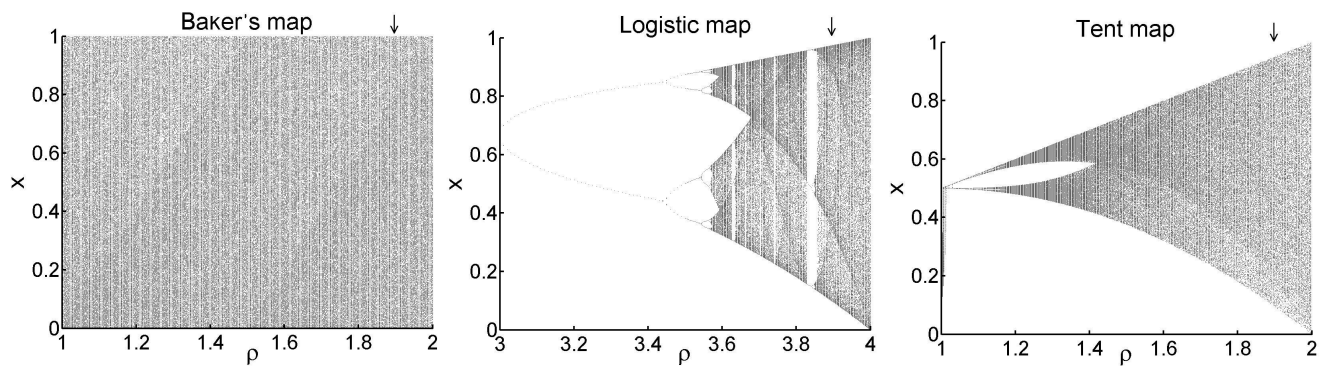

Figure 3. Bifurcation diagrams for the three simulated chaotic maps. To obtain a chaotic series of numbers, well distributed along the output range $[0,1]$, it is necessary to use values for the generating parameter $\rho$ near its limiting value as can be observed for the logistic and tent maps. The baker's map presents good value spreading for all $\rho$ values due to the modulo 1 function, so there is no restriction in its value. Arrows indicate the selected value for each map - 1.9 for baker's and tent maps, and 3.9 for the logistic map.

interesting. In this section, chaotic signals are used to produce the noise resonance. Several chaotic patterns have been generated with one-dimensional maps. Simulations consider three different chaotic maps:

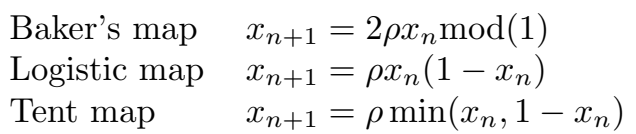

These maps are controlled by parameter $\rho$ that determines their possible values and dynamics. Figure 3 shows bifurcation diagrams for each map. It is interesting to obtain chaotic sequences with as many different values as possible in order to achieve higher resonance peaks. Considering this criteria parameter $\rho$ is selected to drive the maps near their critical point where possible values are maximized (logistic $\rho=3.9$ and tent map $\rho=1.9$ ). The baker's map presents a similar distribution of values in the interval, so any point is valid and $\rho=1.9$ is used.

Chaotic processes have a high sensitivity to initial conditions. This property is used to ensure that the chaotic series for each element are independent. Any initial difference increases with time, producing uncorrelated sequences after a few steps. To study the effect of the different initial points on resonance behavior, 20 simulation runs with different initial values, $x_{0}$, has been carried out. Initial points are spread along its possible range $[0,1]$ at intervals of 0.05 . When the array is simulated, each element receives a different initial value that is equidistant in the corresponding interval of 0.05 . The first 50 points of each sequence are discarded to avoid initial correlation. Figure 4 presents the simulation results for chaotic signals. Lines correspond to the average of the 20 runs. Error bars indicate maximum and minimum points in each noise amplitude. Noise resonance is present in all cases. 

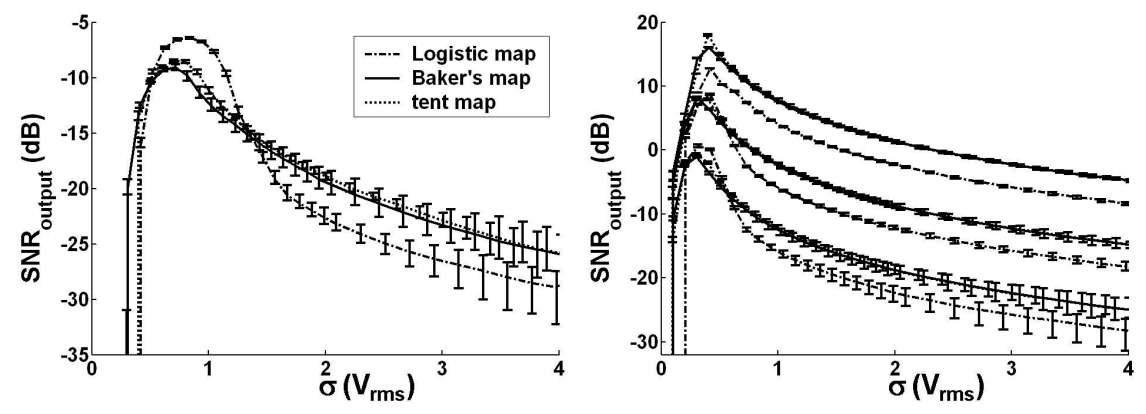

Figure 4. Noise resonance plots for chaotic signals. Simulation setup are equivalent to Fig. 2. Each simulation plot is repeated 20 times with different initial values, $x_{0}$, for chaotic generators, in the range $[0,1]$. Lines indicates the resulting average, and error bars their maximum and minimum values. Differences in pdfs are the reason of the different behavior between the logistic, baker's and tent maps.

Even though the initial setup of chaotic sequences produces some differences, mainly for large noise amplitudes, the resonance value is weakly affected.

Baker's and tent maps have a similar behavior in all structures. The logistic map presents a different noise resonance behavior. In low $N$ systems it has a better performance, while for high $N$ systems the noise resonance value is lower. The reason is that their pdfs are different. A logistic map produces an u-shaped distribution, while the others produce a uniform distribution. So in a logistic map intermediate values are less probable than than extremal values. In low $N$ systems, this results in fewer elements ineffectually switching, thus improving the SNR. However, in high $N$ systems it is necessary to achieve as many different threshold levels as possible and in this situation a u-shaped distribution is clearly inferior compared to a uniform distribution. If chaotic induced noise resonance is compared to stochastic resonance for signals with the same distribution (tent and baker's map versus uniform stochastic distribution) no relevant differences can be observed. Both chaos and stochastic noise appear to produce the same response in SR phenomena, for a threshold-based non-linearity.

\subsection{Periodic perturbations}

Any perturbing noise produces threshold crossings, thus enhancing signal transmission. In this section, simulations with periodic perturbations are investigated. Using these signals, the effects of their characteristics and the conditions necessary to produce the noise resonance phenomenon are outlined. The periodic perturbing signals studied here are square, sawtooth and sinusoidal waveforms with a $1 \mathrm{kHz}$ frequency. In the array system each element receives the same signal, but delayed by a factor $i \cdot 2 \pi / N$.

Figure 5 (left and center) plot the results of these simulations. Noise resonance is produced with delayed signals, as pointed out by Oliaei. ${ }^{19}$ Peak values are higher than those for other noise signals, as these situations are the best. The square waveform has the best response in a single threshold system, but the worst in the array system. This signal can only vary the threshold crossings in two ways. In the noise resonance regime, for the one element system, the input signal offset is canceled producing the best detection situation for a comparator. When the array structure is considered, all elements have either high or low noise added, consequently the average function is useless. For sinusoidal and sawtooth perturbations, noise resonance peaks are similar to other signals. However filtering is more effective, because the power spectrum of periodic noise is separated from signal spectrum. When multiple cells are considered these signals produce the best behavior, as interference signals are perfectly uncorrelated in space and no unnecessary repeated threshold crossings occur in the array. Highest peaks are for the sawtooth perturbation, as it spreads the values for each period of the signal linearly so the system is converted into a flash ADC with perfectly distributed threshold levels when the noise resonance peak is achieved. Using sinusoidal perturbations allows us to study the frequency relationship between the noise and input signal. In fact, sinusoids can be considered as extremely colored noise. Figure 5 right presents the resonance curves for perturbing sinusoids of 3,10,100 and $1000 \mathrm{~Hz}$ for array systems. No significant difference is observed except for little fluctuations by particular situations in each simulation. In single element systems, 

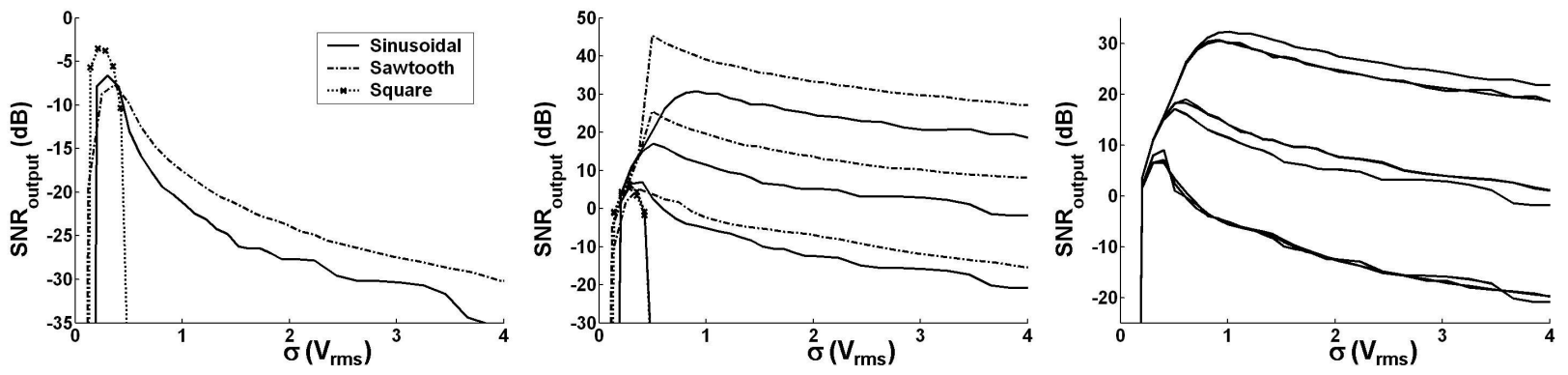

Figure 5. Left and center: Resonance produced by periodic perturbing signals. Perturbing signals are square, sawtooth and sinusoidal waveforms all at $1 \mathrm{kHz}$. In array simulations, each cell $i$ receives the same perturbing signal, but delayed by a factor $i \cdot 2 \pi / N$. Square wave perturbations only provide one extra degree of freedom to the system, so the array structure cannot improve on the single element performance. The other signals present higher noise resonance peaks, as they provide the best possible distribution of threshold crossings. Right: SNR plots for a system with array structure $(N=5,50$ and 500) with perturbing sinusoidal signals of frequencies 3,10,100 and $1000 \mathrm{~Hz}$. The ability to filter signals with equal frequencies is shown.

perturbations with frequencies out of the input bandwidth are needed as no spatial information to remove them is available.

\subsection{Coupled noise in complex systems}

Stochastic and chaotic signals, as well as delayed deterministic signals, produce noise resonance as has been shown. In real complex systems with a large number of elements (such as electronic integrated circuits or, in biology, neurons) parasitic interactions between nearby devices produce noise signals that affect device behavior. In this section, the performance of coupled noise in producing noise resonance is investigated.

To evaluate this kind of noise it is necessary to build a model of the system in order to extract noisy interactions. A model based on a randomly connected cellular automata (CA) has been used - a 2-D CA with $100 \times 100$ cells, with 5 inputs per cell, chosen among the three nearest layers and a common fixed function. Figure 6 left shows the detail of the system. Cell activity depends on neighborhood states and no global communication is present. Noise in each cell is calculated as the superposition of capacitive coupling from cells switching in their environment. The magnitude of the coupling waveforms depends only on the distance between interfering and interfered cells, as the assumption of symmetry in all directions and no delay has been made. Coupling signals have been modeled as an exponential rise and relaxation waveform. Time evolution of the CA is made at discrete time steps. Combining this fact with the duration of coupling waveforms it is possible to roughly model synchronous systems (clock updating is carried out every 4 time steps permitting the relaxation of noise waveforms) or asynchronously (cells update their output values at each time step). Even with this simplified model a very diverse noise values appears - noise signals are captured for rectangular ensembles of $25 \times 20$ cells. For more information in the simulation process see. ${ }^{20}$

Once noise signals are captured they are used to drive threshold devices. Figure 6 (center and right) plots three different cases. Set A calculates noise signals from the switching activity of cells, until distance 2 (two adjacent layers) and in synchronous mode. Set B also considers distance 2, but in the asynchronous regime. Finally Set $\mathrm{C}$ considers cell activity until distance 6 , in asynchronous operation. The noise sequences have a Gaussian-like distribution, so stochastic resonance for normal distribution may be used as a reference. In a single element system, performance in all cases is equal and the noise resonance peak has the same value as in stochastic noise (Fig. 2). In the array system, the differences between each case arise as spatial correlation largely depends on their characteristics. Set B (asynchronous and local) presents the best response as noise differences among nearby cells are larger. Set A (synchronous and local) loses some variability in the signals as changes per unit time are reduced that produce a decrease of SNR. Finally Set C (asynchronous and long range) presents the worst resonance peaks. Increasing the coupling range in a perfectly symmetrical system, without considering delays, largely homogenizes the noise signals reducing the possibility of noise reduction by spatial averaging. In 

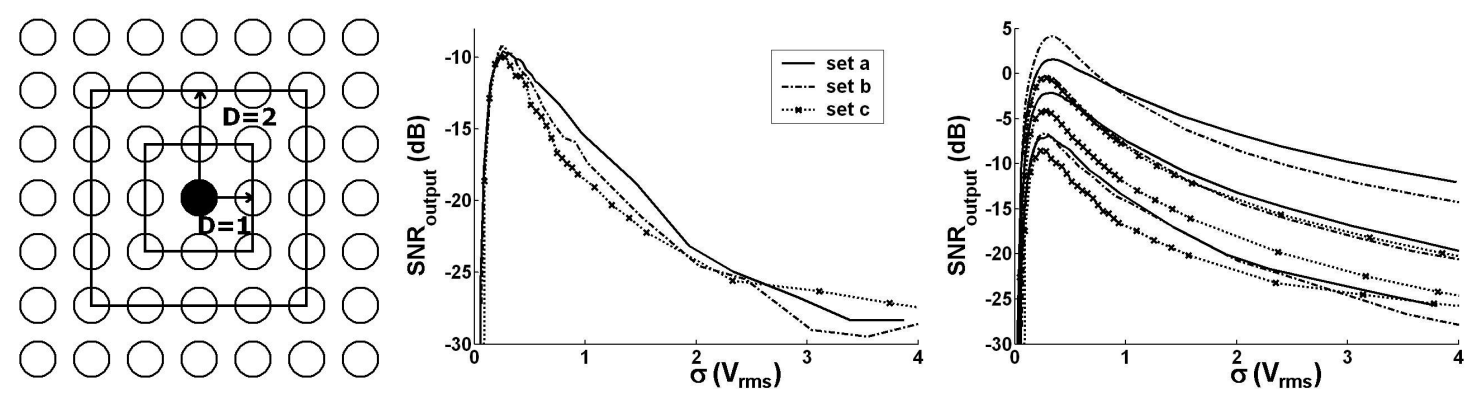

Figure 6. Left: Detail of the 2-D CA showing a sample cell and its possible connecting neighborhood and cells at distance 1 and 2. Center, Right: Resonance curves induced by capacitively coupled noise in complex systems. Noise signals are obtained from randomly connected cellular automata. Three sets of noise signals have been extracted from a CA. Set A simulates a synchronous system with couplings to distance 2, set B and set C emulates asynchronous systems with couplings until distance 2 and 6 respectively. A single threshold element has similar performance with other signals. However an array system presents lower peaks due to spatial correlation of perturbing signals.

all cases, peaks are lower than the Gaussian stochastic noise case. However noise resonance is present and can be improved if cells are separated to increase dispersion in noise values. Also more spatial diversity in noise signals could be attained, if delays due to distance between pairs of cells is considered.

\subsection{Relationship between input and noise amplitude in the noise resonance regime}

In comparing the performance of multithreshold systems versus single threshold arrays ${ }^{12}$ some relevant hints are observed. For low noise amplitudes (related to input signal) a multithreshold array provides better results as its resolution does not depend on noise. For large noise amplitudes the advantage provided by multiple thresholds is lost as noise shifts the effective signal at any level and both systems have a similar performance. Finally, in noise resonance, for mid noise amplitudes, the single threshold system is able to outperform the more complicated multilevel system. It is convenient to work in the noise resonance regime to obtain the best results from the system. This section outlines the relation that should arise between input signal and noise to keep the system in stochastic resonance regime. The simulations presented consider stochastic uniform noise, but no significant differences arise for other noise signals.

Two sets of simulations have been studied. The first one considers a sinusoidal input wave with offset $0 \mathrm{~V}$ and amplitudes from 0.01 to $1 \mathrm{~V}$. The second set uses a sinusoid with fixed amplitude $(0.1 \mathrm{~V})$ and variable offset from 0 to $1 \mathrm{~V}$ - negative values have a symmetrical response. In each case noise amplitude, output SNR and theoretical maximum output value* in noise resonance are extracted and plotted in Figure 7. In both simulations systems with $N=1,5,50$ and 500 have been considered. A single threshold situation with zero input offset signal cannot improve information transference, so maximum SNR corresponds to no noise. This case has been obviated for being trivial.

Maximum SNR appears when signal power is maximized and noise power minimized. Noise resonance peaks occur when both conditions are fulfilled. Figure $7 \mathrm{~b}$ shows that resonance fully expands input range into output maximizing signal power. It is achieved by maintaining a 1:1 relation between signal and noise amplitudes at the input (Fig. 7a) that force maximum output at its full range. Minimization of noise is achieved by overexpanding the signal into the output range when a symmetrical situation appears (amplitude variation for $N=5$ with maximum output at $1.6 \mathrm{~V}$ ) so noisy transitions in the largest values are cut by system saturation without losing signal strength. At resonance point, SNR only depends on the system's structure, as signal power is related to output levels and noise power to number of devices in input array. Fig. 7c illustrates this. Offset variation has a dependence with its value because the dc component has not been considered in SNR integration.

${ }^{*}$ As seen in section 2.2 the system converts the input range defined by noise into the output range determined by output levels through a function related to noise pdf. From Equation (7) it is possible to define a gain factor between input and output signals $(D / 2 A)$. This value is used to calculate the maximum output signal at noise resonance point, given a maximum value of input signal and noise amplitude. 

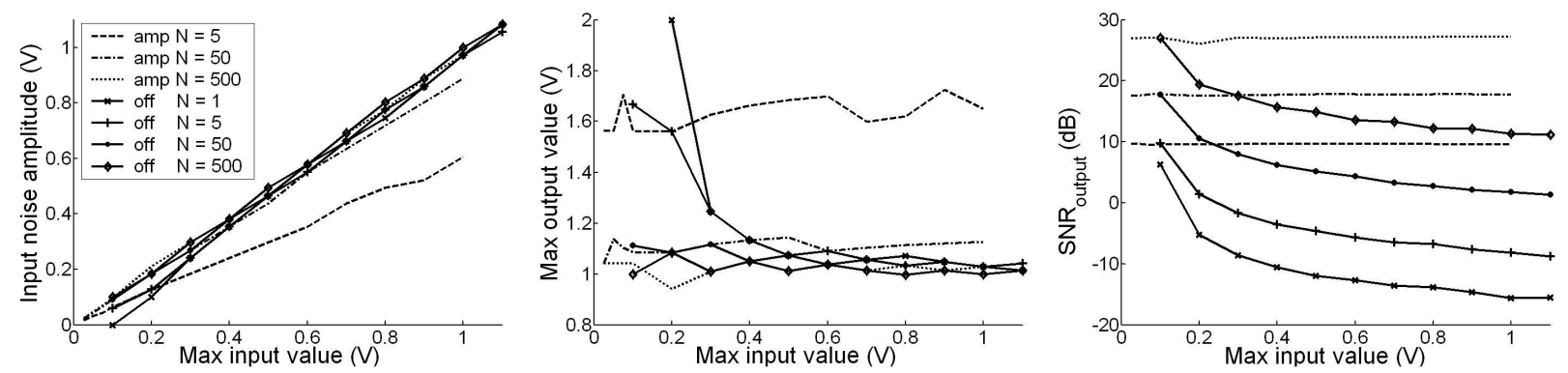

Figure 7. From left to right: a) noise amplitude, b) theoretical maximum output value (gain · input max $_{\text {) }}$ and c) output SNR in the resonance point for several amplitudes and offsets of the input signal. The input signal is a sinusoidal wave with frequency $10 \mathrm{~Hz}$. The set of curves marked 'amp' use a $0 \mathrm{~V}$ offset, with amplitudes from 0.001 to $1 \mathrm{~V}$ and the set marked 'off,' a $0.1 \mathrm{~V}$ amplitude with offset from 0 to $1 \mathrm{~V}$. Plots show that a fixed relation is present between input and noise amplitudes, suggesting a rule for keeping within the noise resonance regime.

To force a noise resonance regime, the system should maintain the maximum output level just in the limit of saturation of its output range. This can be done either controlling noise amplitude or input signal strength.

\section{NON-IDEAL THRESHOLD DEVICES}

So far simulations and analysis have considered ideal comparators with infinite slope, zero delay and hysteresis and perfectly matched threshold values. In this section we will show that noise resonance is robust to device non-ideal characteristics. This fact will permit to use noise resonance in real devices as electronic circuits. Simulations check the effects of main non-ideal problems appearing in real threshold devices. The perturbing signal used in simulations is a stochastic uniformly distributed noise. No qualitative differences occur for other signals as previous derivation have shown. The values used for the deviations of the ideal function have been selected to have significance in front of input signal range not to provide real circuit characteristics.

\subsection{Threshold error}

Process variations induce mismatch between devices. One of the critical values for the system is the threshold level. In single device systems, threshold fluctuations only induce an offset error in the output signal. In array systems, the effect is also an effective threshold error (mean of all offsets) but if fluctuations are big enough to spread the values along the input signal extension, the system loses the resonance peak as it is converted in an array of randomly perturbed comparators. In the following simulations, fluctuations in threshold value have been modeled with a Gaussian distribution for a given standard deviation. To obtain statistics of the effects on noise resonance, the simulation creates 100 sets of thresholds and simulates the system for each of them. Figure 8 presents these results with the mean value of all runs and their maximum and minimum values. The input signal range is from -0.15 to $-0.65 \mathrm{~V}$. In the worst case, $\sigma=0.5$, thresholds can be sufficiently distributed over the input signal to avoid noise resonance behavior. However, minimum values still present it. For lower standard deviations of threshold distribution, noise resonance is not affected. The effects of threshold errors are very pronounced for low noise runs, however from the noise resonance peak to high noise its importance is reduced by the effect of the noise.

\subsection{Hysteresis}

Systems usually are not completely symmetrical and real threshold devices often have a hysteresis either due to parasitic effects or by design to avoid oscillations. In the following simulations the effect of this behavior on noise resonance is outlined. Hysteresis can be symmetrical with respect to the ideal threshold value or more generally asymmetrical. Figure 9 plots the noise resonance curves for symmetrical hysteresis. Resonance is maintained with few changes, except an increase in noise amplitude as the effective threshold to produce a level crossing is increased by hysteresis width. In the asymmetrical situation the effect is equivalent. When the signal 

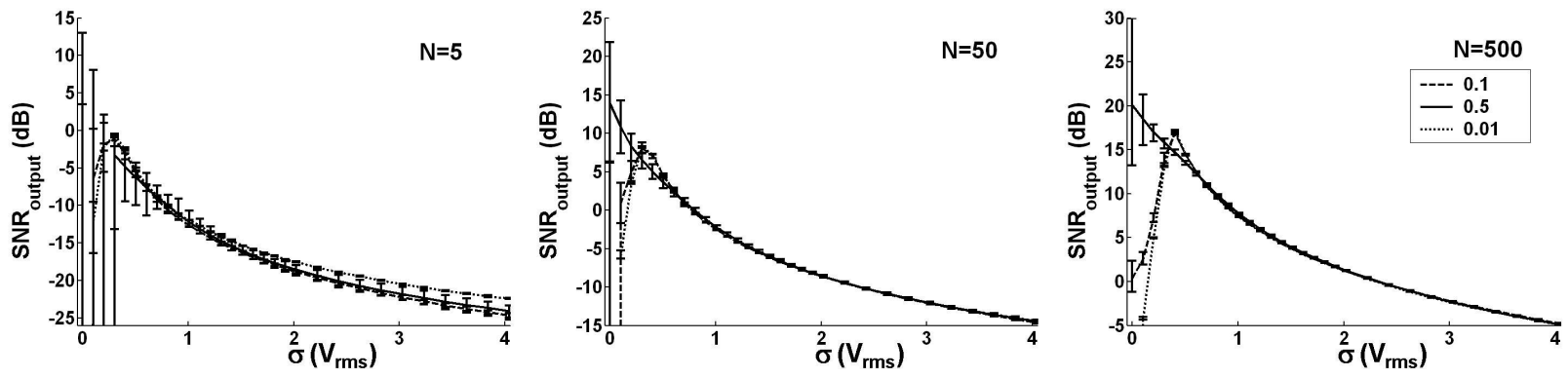

Figure 8. Effects of dispersion in threshold values for array system with 5, 50 and 500 elements. Threshold values have been assigned according to a Gaussian distribution with $\sigma=0.5,0.1$ and 0.01 . For each point 100 runs with different sets of comparison levels have been realized. Plots show mean, maximum and minimum values at each point. Resonance is lost if threshold values are spread over the input signal range.
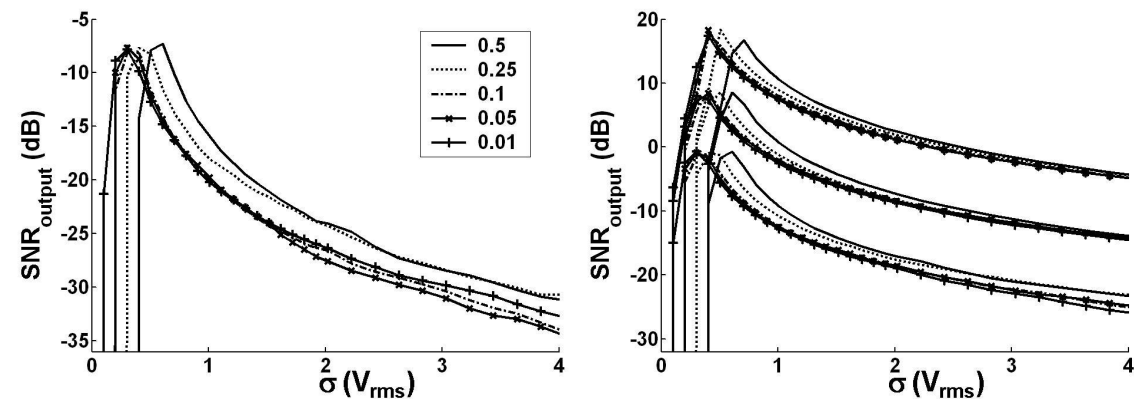

Figure 9. Effects of noise resonance by considering symmetric hysteresis in threshold elements with values $0.5,0.25,0.1$, 0.05 and $0.01 \mathrm{~V}$. Simulation for single element and array structure are plotted showing the same response: an increase in noise amplitude to achieve the resonance point.

is subthreshold, low to high hysteresis width determines the extra noise in resonance and vice versa. Another difference is that, in this situation, amplitudes of the composed signal (noise plus input) should be bigger than the total hysteresis width to obtain any information at the output, so hysteresis limits the minimum signal that can be detected by the system.

\subsection{Finite gain}

Ideal comparators have an infinite slope when changing between levels. This abrupt change is not possible in real implementations as it implies instantaneous switching in which infinite energy was necessary. So threshold devices have a certain gain in the transition point. Assuming that threshold value is set at the zero crossing, comparator gain determines a symmetrical area before and after the threshold in which device behaves linearly. Figure 10 depicts a set of simulations with comparator gains from 2 to 1000 (with associated linear sections from 1 to $0.002 \mathrm{~V}$ respectively). In single element systems, when the linear region reaches the input values part of the signal passes to the output. In this case the zero noise point has the maximum SNR value. If this situation is not present, the resonance is not affected. In an array system, resonance is always present. Even if the linear region reaches the input signal, the zero noise point contains information but due to the combination of output signals (averaging function) several points with non-zero noise have better SNR.

\subsection{Device response time}

Another important property of real systems is response time. All real systems are causal and have delays that should be considered. The response time of comparators defines input-output delay and also the minimum time a signal should be above or below the threshold to produce a transition at the output. This time will determine the maximum frequency component of the perturbing signal that can be used to produce noise switching. The 

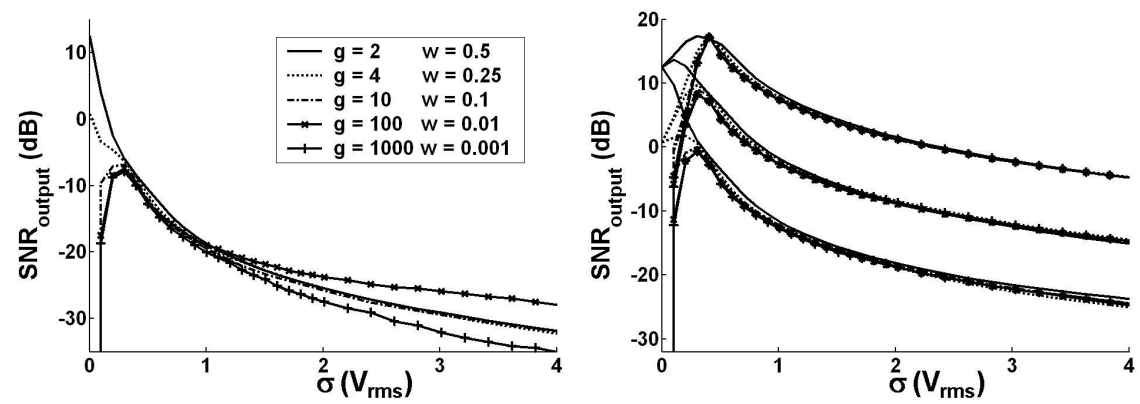

Figure 10. The system performance when threshold elements with finite gain $(2,4,10,100$ and 1000) are considered. Simulations show that gain eliminates the noise resonance in the single thresholding element, when the width of linear section permits that input signal information propagates to the output. In all other situations improvement with noise is present.
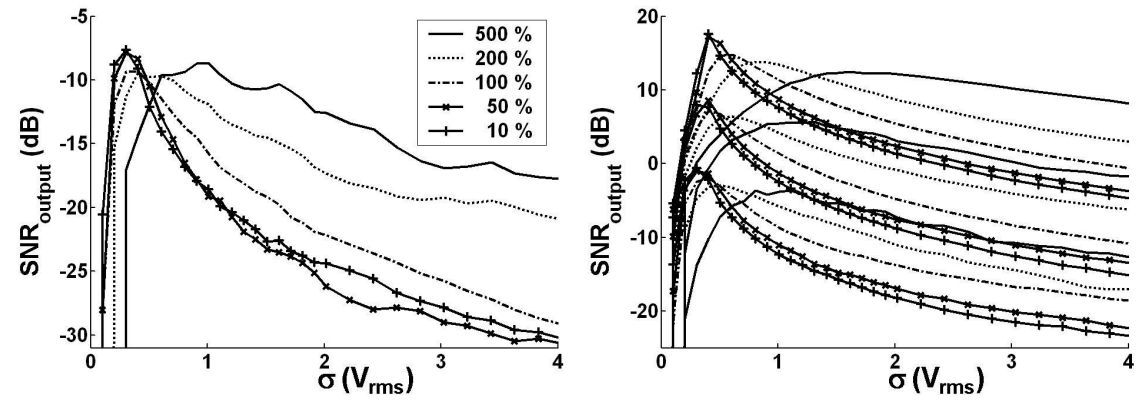

Figure 11. Effect of response time of devices in noise resonance. Response time values are taken in relation to the simulation step $\Delta t$ to permit a broader significance of the results. Each device requires that its input signal, $S_{i}(t)$, remains the indicated percentage of $\Delta t$ on one side of its threshold level to a change at its output. The effect is a low-pass filtering producing an increase of noise amplitude in noise resonance and a higher noise reduction for large noise amplitudes.

overall effect is low-pass filtering realized by the comparator, when high frequency noise (related to its response time) is present.

Due to methods used to obtain stochastic and chaotic series and the simulation scheme, time values for noise signals have little significance. What is interesting to observe is the effect, which signals faster than device response time produce, on noise resonance. To obtain results independent simulation configuration, response time has been defined in relation to $\Delta t$. So the result is valid for any relation of real response time and noise bandwidth. Figure 11 shows the simulation results for several response times - values from $5 / \mathrm{BW}_{\text {noise }}$ to $0.1 / \mathrm{BW}_{\text {noise. }}$. When the response time is under $1 / \mathrm{BW}_{\text {noise }}$ effects on resonance are negligible. For higher delays, noise resonance is present but noise is filtered. The resulting effect is an improvement of SNR for high amplitude noise and the need of extra noise amplitude (its effective value is reduced) to produce the resonance. The peak value is reduced because the number of crossings is decreased and less input information can arrive at the system's output.

\section{CONCLUSIONS}

A simple mathematical analysis of threshold systems is presented. It determines that only the pdf of perturbing signals and the input waveform defines the system output. We demonstrate that the phenomena of SR and SSR are valid for stochastic, chaotic, deterministic and capacitively coupled noise through direct simulation - thus extending stochastic resonance to a more general noise resonance concept. Comparison of noise resonance for all cases agrees with the analysis provided. There are no differences between different noise sources, only their 
pdfs are important. The relationship between input and noise amplitudes to force systems to work in the noise resonance regime has been outlined, so optimum response can be achieved. Finally the robustness of SR and SSR to non-ideal characteristics of threshold devices (i.e. threshold error, hysteresis, finite gain and response time) has also been demonstrated. The combination of all three facts opens up the the possibility of applying SR concepts to electronic circuit design.

\section{ACKNOWLEDGMENTS}

This work has been supported by the grant AP2002-2600 and the project CYCIT TIC 2001-2337. Funding from GTECH Australasia is also gratefully acknowledged.

\section{REFERENCES}

1. J. K. Douglass, L. Wilkens, E. Pantazelou, and F. Moss, "Noise enhancement of information transfer in crayfish mechanoreceptors by stochastic resonance," Nature 365, pp. 337-340, 1993.

2. J. Levin and J. Miller, "Broadband neural encoding in the cricket cercal sensory system enhanced by stochastic resonance," Nature 380, pp. 165-168, 1996.

3. M. DeWeese and W. Bialek, "Information flow in sensory neurons," Il Nuovo Cimento 17D, pp. 733-741, 1995.

4. X. Yu and E. Lewis, "Studies with spike initiators: linearization by noise allows continuous signal modulation in neural networks," IEEE Trans. on Biomedical Eng. 36(1), pp. 36-43, 1989.

5. B. Ando and S. Graziani, "Adding noise to improve measurement," IEEE Instr. and Meas. Magazine 4(1), pp. 24-31, 2001.

6. R. Stewart and E. Pfann, "Oversampling and sigma-delta strategies for data conversion," Elec. and Com. Eng. Jour. 10(1), pp. 37-47, 1998.

7. S. Meninger, J. Mur-Miranda, R. Amirtharajah, A. Chandrakasan, and J. Lang, "Vibration-to-electric energy conversion," IEEE Trans. on VLSI Systems 9(1), pp. 64-76, 2001.

8. I. Lee, X. Liu, B. Kosko, and C. Zhou, "Nanosignal processing: stochastic resonance in carbon nanotubes that detect subthreshold signals," Nano Letters 3(12), pp. 1683-1686, 2003.

9. Z. Gingl, L. B. Kiss, and F. Moss, "Non-dynamical stochastic resonance: Theory and experiments with white and arbitrarily colored noise," Europhys. Lett 29(3), pp. 191-196, 1995.

10. B. McNamara, K. Wiesenfeld, and R. Roy, "Observation of stochastic resonance in a ring laser," Phys. Rev. Lett. 60, pp. 2626-2629, 1988.

11. R. Benzi, A. Sutera, and A. Vulpiani, "The mechanism of stochastic resonance," Jour. Phys. A 14, pp. 453457, 1981.

12. N. Stocks, "Suprathreshold stochastic resonance in multilevel threshold systems," Phys. Rev. Lett. 84(11), pp. 2310-2313, 2000.

13. D. Williams and D. Johnson, "Narrowband array processing algorithms for arbitrary noise distributions," Int. Conf. on Acoustics, Speech, and Signal Processing 4, pp. 2830-2833, 1989.

14. L. Gammaitoni, P. Hanggi, P. Jung, and F. Marchesoni, "Stochastic resonance," Reviews of Modern Physics 70(1), pp. 223-287, 1998.

15. B. Kosko and S. Mitaim, "Robust stochastic resonance: signal detection and adaptation in impulsive noise," Physical Review E 64, 051110, 2001.

16. M. D. McDonnell, C. E. M. Pearce, and D. Abbott, "A characterization of suprathreshold stochastic resonance in an array of comparators by correlation coefficient," Fluctuation and Noise Letters 2(3), pp. L205L220, 2002.

17. C. A. Skarda and W. J. Freeman, "How brains make chaos in order to make sense of the world," Behavioral and Brain Sciences 10, pp. 161-195, 1987.

18. M. Bucolo, R. Caponetto, L. Fortuna, M. Frasca, and A. Rizzo, "Does chaos work better than noise?," Elec. and Com. Eng. Jour. 2(3), pp. 4-19, 2002.

19. O. Oliaei, "Stochastic resonance in sigma-delta modulators," Electronics Letters 39(2), pp. 173-174, 2003.

20. F. Martorell and A. Rubio, Unpublished results, 2004. 